\title{
TEMPORAL STABILITY OF SUNSPOT UMBRAL INTENSITIES: 1986-2012
}

\author{
G. De Toma ${ }^{1}$, G. A. Chapman ${ }^{2}$, A. M. CoOKSON ${ }^{2}$, And D. Preminger ${ }^{2}$ \\ ${ }^{1}$ High Altitude Observatory, National Center for Atmospheric Research, P.O. Box 3000, Boulder, CO 80301, USA; detoma@ucar.edu \\ ${ }^{2}$ San Fernando Observatory, Department of Physics and Astronomy, California State University Northridge, Northridge, CA 91330, USA \\ Received 2013 May 15; accepted 2013 June 6; published 2013 June 20
}

\begin{abstract}
We examine the relative intensity of sunspot umbrae during the period from 1986 to 2012 using photometric images from the San Fernando Observatory. We confirm the presence of a relationship between the mean umbral core intensity and the mean sunspot area, as found in previous studies, and do not find a notable change in this relationship between cycles 22 and 23. We looked for a possible time variation in the sunspot umbral contrast during the $27 \mathrm{yr}$ covering cycles 22,23 , and the rise of cycle 24 , and we did not find a significant change. These findings do not indicate that sunspots have become less dark during cycles 23 and 24.
\end{abstract}

Key words: Sun: activity - Sun: photosphere - sunspots - Sun: surface magnetism

Online-only material: color figures

\section{INTRODUCTION}

In order to understand solar variability over long periods of time and to decipher the workings of the solar dynamo, it is important to know whether the magnetic field in sunspots exhibits any secular trend.

The determination of magnetic field strength in spot umbrae is challenging and can be accurately obtained only with spectropolarimetry or in lines where the Zeeman wavelength splitting can be directly measured (e.g., Penn \& Livingston 2006). These kinds of measurements have typically been obtained in the past only for a limited number of cases. Synoptic programs that measure the line-of-sight component of the magnetic field have been available for years or decades from the ground, for example, at the National Solar Observatory, and from the Michelson Doppler Imager (MDI) on the Solar and Heliospheric Observatory satellite, but they only provide magnetic flux density and are limited by the assumption that the magnetic field is radial and by saturation effects in spot umbrae.

Measures of magnetic field strength and continuum intensity in the darkest part of the spot umbra have been obtained with the McMath-Pierce telescope at Kitt Peak since 1998, using the IR Fe I line at $1565 \mathrm{~nm}$ that allows for a very accurate determination of the magnetic field strength. Penn \& Livingston (2006) analyzed these observations and reported that the magnetic field strength in sunspots was decreasing, independently from the solar cycle. A linear fit to these data gave a decrease of about $50 \mathrm{G} \mathrm{yr}^{-1}$ and a corresponding increase in intensity of about $1.8 \% \mathrm{yr}^{-1}$. They concluded that, if this rate of change continued, sunspots would have vanished by 2015 (Livingston et al. 2009). Their most recent analysis which includes up-todate data (Livingston et al. 2012) show a smaller decrease of about $46 \mathrm{G} \mathrm{yr}^{-1}$. If such a decrease continues, they predict there will be almost no spots in cycle 25 .

Initially, sunspot spectra were acquired with the McMath-Pierce once a month over a period of two or three consecutive days and using a limited number of selected spots each day (Penn \& Livingston 2006). These early observations focused on large spots. In recent years, as more observing time was allocated to the program, the frequency of the observations has improved with almost daily observations acquired at present. In addition, a broader range of spot sizes has been in- cluded over time. Currently, spots of all sizes, including pores, are observed (Penn \& Livingston 2010). The result is a nonhomogeneous data set in time coverage and sunspot sampling. Nonetheless, this is a valuable and very long record of accurate sunspot umbral magnetic field measures.

The disappearance of sunspots speculated by Livingston et al. (2012) has important implications and indicates that the Sun would go into a new Maunder Minimum in the near future. However, this strong decline in sunspot magnetic field strength has not been confirmed by other magnetic field observations and remains controversial. IR spectropolarimetric measurements of magnetic field strength and umbral intensity taken between 1999 and 2011 (Rezaei et al. 2012) and the long record of magnetic field measurements from Russian observatories (Pevtsov et al. 2011) did not show a decline in magnetic fields with time but rather a solar cycle dependence. These observations indicate that spots have, on average, weaker fields and brighter umbrae near solar minimum, which is consistent with the scarcity of large spots during times of low activity.

The intensity of sunspot umbrae, and particularly the intensity in the darkest part of the umbra, has been found to be a good proxy for the strength of the magnetic field in spots (e.g., Norton \& Gilman 2004; Schad \& Penn 2010 and references therein). Norton \& Gilman (2004) demonstrated that the umbral peak magnetic field strength is inversely proportional to the minimum umbral intensity divided by the nearby quiet-Sun continuum intensity. They showed that MDI umbral intensity gave a more reliable estimate of the strength of magnetic fields in spots than MDI magnetic fluxes. A linear relationship between continuum intensity and magnetic fields was later confirmed by Schad \& Penn (2010). The McMath-Pierce observations also show a clear correlation between these two quantities. For these reasons, umbral intensities can be used to verify the Livingston et al. (2012) results. Analyses of spot umbrae have been performed using different data sources. They have shown either no significant variation with the solar cycle of umbral intensity (Mathew et al. 2007; Wesolowski et al. 2008) or a weak solar cycle dependence (Norton \& Gilman 2004; Penn \& MacDonald 2007; Schad \& Penn 2010), but no clear long-term trend.

In this Letter, we study spot umbrae using a large sunspot data set obtained at the San Fernando Observatory (SFO) from 1986 to 2012. We check if long-term changes can be detected in 
umbral intensities, and specifically if spot umbrae are becoming less dark with time. In Section 2, we briefly describe the SFO observations used in our study. In Section 3, we analyze the umbral core intensities as a function of spot area and time. In Section 4, we provide a possible explanation for the results obtained by Penn \& Livingston (2006). Conclusions are given in Section 5.

\section{OBSERVATIONS}

We use photometric data obtained at SFO since 1986 with the Cartesian Full Disk Telescope 1 (CFDT1) at $672.3 \mathrm{~nm}$ with a $10 \mathrm{~nm}$ bandpass and a spatial resolution of about $5^{\prime \prime}$ pixel $^{-1}$ (Chapman 2001, 2004). Our study extends a similar analysis back and forward in time (Wesolowski et al. 2008) based on data obtained with CFDT2, a higher resolution telescope operated at SFO since 1992. The CFDT1 data set, in spite of its low spatial resolution, has the advantage of covering both solar cycle 22 and 23 with the same instrument and is largely unaffected by seeing conditions.

At SFO, spots are automatically identified based on their photometric contrast from calibrated images that are corrected for limb darkening (e.g., Chapman 1992; Preminger et al. 2001). The per-pixel photometric accuracy of the contrast images is better than $1 \%$ (Walton et al. 1998). Sunspots are defined as those pixels that are at least $8.5 \%$ darker than the quiet Sun, and spot umbrae as the pixels that are at least $32 \%$ darker. The data used in our analysis include spot area and spot minimum intensity measured in the umbral core.

The CFDT1 images used here were not restored to correct for scattered light. At the CFDT1 resolution, the main consequence of scattered light is a measured contrast lower than the real sunspot contrast but values before and after restoration are well correlated, except for the smallest spots, for $\mu$ values greater than 0.7 (where $\mu$ is the cosine of the heliocentric angle), as shown in Figure 4 of Walton \& Preminger (1999). To minimize projection effects near the limb and scattered light contamination, we limit our analysis to $\mu$ larger than 0.7 , which corresponds to spots located within $0.71 R_{\odot}$ from disk center. This reduces the number of data points in the SFO record from 32,545 to 22,765 over the $27 \mathrm{yr}$ period from 1986 to 2012, which still gives excellent statistics and very good coverage of spot sizes.

\section{SUNSPOT PROPERTIES}

First, we examined the umbral relative intensity $I_{\text {umbra }} / I_{\text {quiet Sun }}$ as a function of the spot area for the entire SFO data set. Figure 1 shows the relative intensity computed in the darkest pixel of the spot (umbral core) as a function of spot area for all the spots for which $\mu$ is larger than 0.7 . Intensity can vary greatly for a given spot area but when data are averaged based on the spot size, as shown in the bottom panel of Figure 1, a clear relationship is found between umbral core intensity and spot area in both cycles. Larger spots, on average, are darker than smaller spots, as found in other studies. The darkness of the umbra increases with spot area up to areas of about $1000 \mu$ hem (where $\mu$ hem indicates millionths of solar hemisphere) and then tends to approach an asymptotic value. Because very large spots are infrequent, only a few observations are available for spots with areas larger than $1000 \mu \mathrm{hem}$. We have used increasingly larger area bins for larger areas to improve the statistics, but the number of data points is smaller for the largest spots.

We find that small to medium spots with area between 100 and $400 \mu$ hem and large spots with area between 900 and
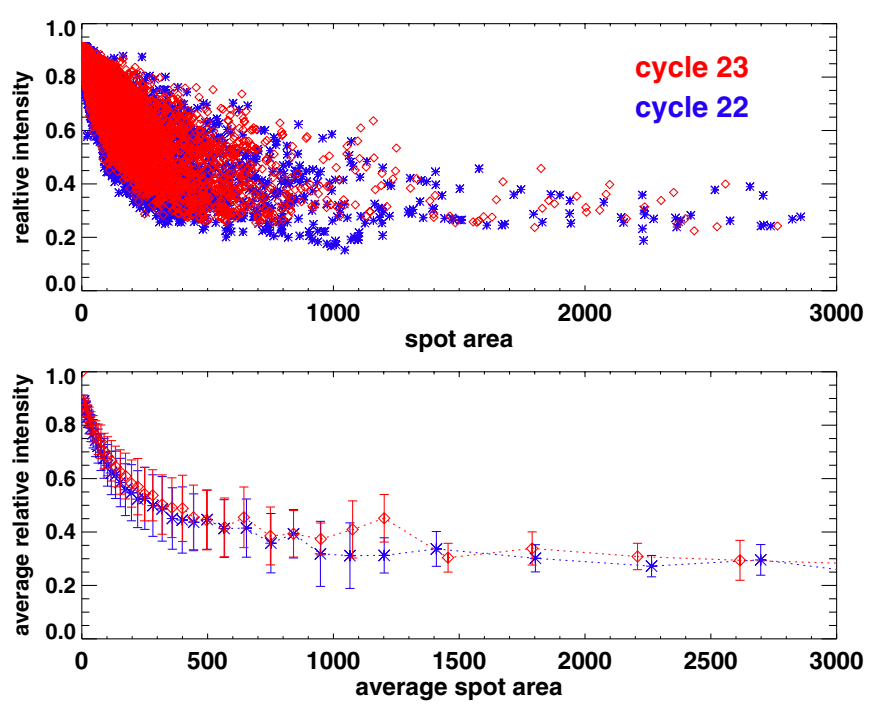

Figure 1. Relative intensity measured in the darkest pixel of the spot umbra as a function of spot area for all SFO sunspot observations with $\mu>0.7$ (top panel). Average umbral core intensity as a function of spot area (bottom panel). The error bars correspond to $\pm 1 \sigma$, where $\sigma$ is equal to the rms of the intensities in each area bin.

(A color version of this figure is available in the online journal.)

$1200 \mu$ hem are slightly less dark in cycle 23 than in cycle 22, but the differences, which are usually $10 \%$ or less, are much smaller than the error due to the scatter in the intensity-area relationship. No systematic differences are found in the two cycles for other spot sizes. Overall, there is excellent agreement within the error bars between the two cycles. Thus, we conclude that the relationship between the umbral core intensity, measured in the darkest part of the umbra, and spot area has not changed within the two cycles. Spot intensity does not appear to be different in cycle 23 .

We then analyzed spot intensities as a function of time. We looked for variations in the umbral core intensity as well as in the mean spot intensity. The top panel of Figure 2 shows the umbral intensity measured in the darkest pixel of each spots for all the spots in the SFO data set for which $\mu$ is larger than 0.7 . A large daily variation is seen throughout the solar cycle with a tendency toward larger intensities during time of low activity, when large spots are rare or absent. The lower envelope of the data points (corresponding to the largest spot present on the Sun at any given time) shows a solar cycle shape in agreement with the results of Nagovitsyn et al. (2012). A first- and second-order fit to the data is overplotted. The first-order fit shows a small increase over the $27 \mathrm{yr}$ of observations of about $3.5 \%$ for the umbral core intensity. This small variation appears to be related to the presence of a few large and very dark spots in the early rise of cycle 22 (de Toma et al. 2013). If only cycle 23 data are fitted, we find a negligible variation of about $1 \%$. Similar fits to mean spot intensities show no significant variation from 1986 to 2012 .

The middle panel of Figure 2 shows the average umbral core intensity computed over 27 days. The 27 day averages show larger fluctuations near solar minimum because of the lower number of spots present on the Sun during this time. To avoid excessive noise due to poor statistics at times of low solar activity, we included only those 27 day intervals when a minimum of 7 spots were observed. A first-order fit is overplotted (dashed line) together with a line at a constant value, corresponding to the mean umbral core intensity of all data 

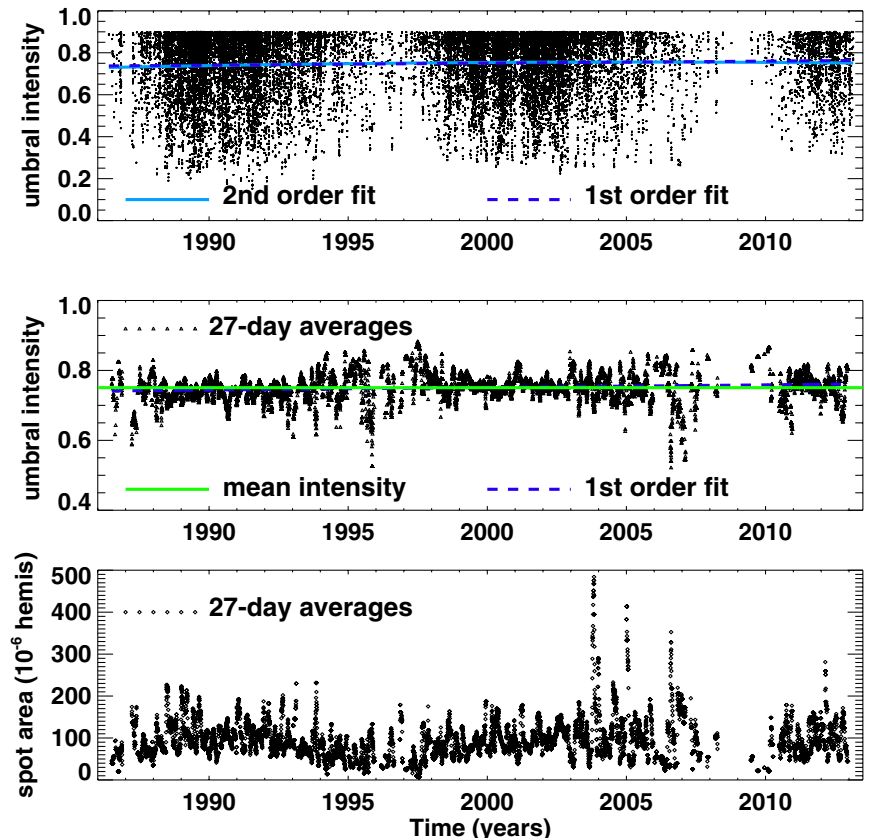

Figure 2. Spot relative intensity measured in the darkest pixel of every spot in the SFO data set with $\mu>0.7$ (top panel). Averages over 27 days for the umbral core intensity (middle panel) and sunspot area (bottom panel). The averages were computed only if at least 7 spots were observed during the 27 day period. (A color version of this figure is available in the online journal.)

(continuous line). The two lines are almost indistinguishable. There is clearly not an upward trend in the umbral core intensity over the $27 \mathrm{yr}$ period, and especially during cycle 23. This result disagrees with the findings of Penn \& Livingston (2006) that reported a change of almost $2 \%$ a year in spot brightness for the period 1998-2006. SFO data show less than 3\% change over the entire period 1986-2012 for 27 day averaged umbral core intensity.

To test the robustness of our result, we selected spots within different distances from the disk center. We repeated the fits using only spots located at $\mu$ larger than $0.5,0.6,0.8,0.9$, and 0.94 . The results remained unchanged in all cases and no notable trend was found during solar cycles 22 and 23 or the rise of cycle 24 . We also tested the effect of excluding the smaller spots with an area less than or equal to 20,40, 60, and $80 \mu \mathrm{hem}$. The removal of small spots revealed a small difference between cycles 22 and 23, which was consistent with the decrease of large spots in cycle 23 found in our previous work (de Toma et al. 2013), but did not significantly alter the results for cycle 23 . We could not detect a systematic increase in spot umbral brightness over cycle 23 .

In summary, we found no evidence that sunspots have been getting less dark during cycle 23 and did not find a solar cycle dependence in the umbral core intensity and mean spot intensity when all spot sizes were included. There was instead a clear solar cycle modulation in the average sunspot area, as shown in the bottom panel of Figure 2. These results are in agreement with those found in previous studies of umbral intensity covering a much shorter time span (Wesolowski et al. 2008; Mathew et al. 2007). We should note that the SFO umbral contrast data shown in Wesolowski et al. (2008) have been incorrectly plotted in Figure 5 of Schad \& Penn (2010). The SFO values are consistent with umbral intensities reported in other papers (e.g., Mathew et al. 2007; Schad \& Penn 2010).
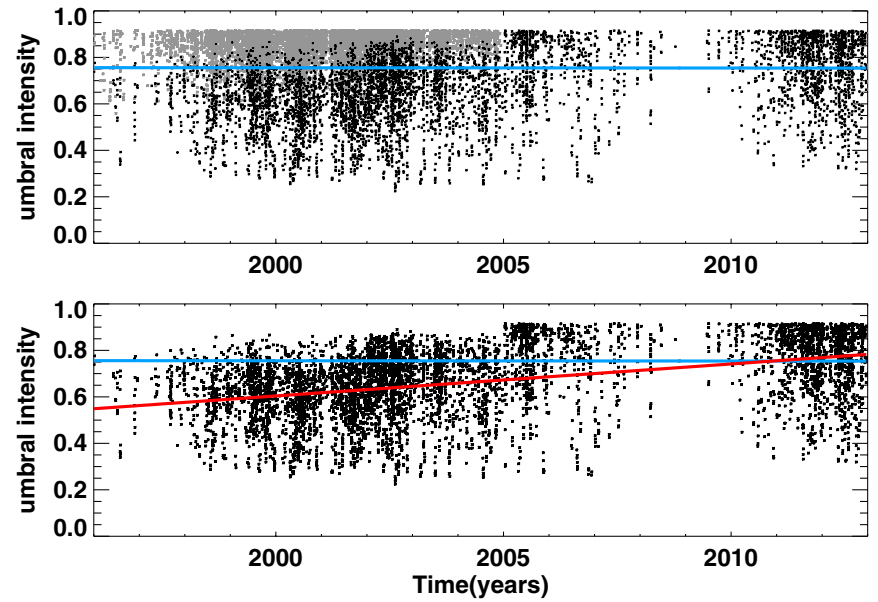

Figure 3. Relative intensity measured in the spot umbral core at SFO during cycle 23 and the rise of cycle 24 . Data points in gray correspond to the small spots that were removed to simulate a selection effect similar to the one present in the McMath-Pierce data. A first-order fit to all data is shown in the top panel. The filtered data and the corresponding first-order fit are shown in the bottom panel. For comparison, we overplot the fit to all data.

(A color version of this figure is available in the online journal.)

\section{DISCUSSION}

It is well known that there is a lower limit to the magnetic field strength associated with a pore (e.g., Leka \& Skumanich 1998). This lower value for the IR Fe I at $1565 \mathrm{~nm}$ corresponds to about $1500 \mathrm{G}$, as discussed by Penn \& Livingston (2010). They find that small spots and pores usually have magnetic fields between 1500 and $2000 \mathrm{G}$. It is interesting to note that, as shown by Figure 2 of Livingston et al. (2012), these weaker fields are not present in their early measurements and become increasingly more frequent in the later data. In particular, umbral magnetic fields of the order of $2000 \mathrm{G}$ start to be recorded in about 2002, magnetic fields lower than $1700 \mathrm{G}$ in about 2004, and magnetic fields as low as $1500 \mathrm{G}$ are only seen after 2011 , when the overall frequency of their measurements becomes high. Correspondingly, low-intensity umbrae are only visible in their data set in later years. Because we know that small spots and pores with umbral magnetic fields of the order of 1500-2000 G are always present on the Sun, the lack of magnetic field strengths within this range in their earlier data indicates that weaker/smaller spots were not observed. Thus, not only were a small number of spots observed at McMath-Pierce in 1998-2002, but these spots were mostly large spots.

The fact that the sparse data points at the beginning of their data set consist mainly of large spots with strong fields and low intensities can significantly bias the result of a linear fit to the data. To examine the consequence of a similar selection effect, and to better compare the SFO measures with the McMath-Pierce measures, we performed the following test. First, we fitted all SFO data from 1996 to the end of 2012. Then, we removed all small spots with area less than $80 \mu$ hem from 1996 to 2001, and all spots smaller than $40 \mu$ hem from 2002 to 2004, and fitted the data gain. The results are given in Figure 3. The top panel shows all SFO data for the period 1996-2012 and the corresponding first-order fit. The gray points correspond to the small spots that were removed in the filtered data. The bottom panel shows the filtered data and the corresponding firstorder fit. Removing the small spots in the early years of cycle 23 introduces a clear upward trend but no trend exists when all spots are included. 
This suggests that the increase in relative intensity of spot umbrae with time, and the corresponding decrease in magnetic field strength, reported by Livingston et al. (2012) are not due to a physical change in the spots but are rather a consequence of the exclusion of small spots and pores in their early measurements. We point out that there is no clear upward trend notable in their data from 2005 to 2012 . Because this is the time period during which a broader range of spot sizes was measured, it further supports the hypothesis that the trend in their data is caused by the dominance of large spots in the early measurements.

\section{CONCLUSIONS}

We examined the relative intensity in sunspot umbrae using sunspot observations taken at SFO with the CFDT1 telescope from 1986 to 2012, covering cycles 22 and 23 and the rise of cycle 24 . The sunspot data set from CFDT1 has relatively low resolution but has the important advantage of being a long and consistent record from a single instrument where sunspots are objectively identified using a contrast criterion.

We find that the umbral intensity can vary greatly for a given sunspot area but, when averaged values are considered, a relationship between the spot area and umbral core intensity becomes clear. We confirm, as found in previous studies, that larger spots have darker umbrae than smaller spots. The minimum umbral intensity, on average, decreases with sunspot area and approaches an asymptotic value for the largest spots that have area greater than $1000 \mu \mathrm{hem}$. When data are analyzed separately for cycles 22 and 23, we find that the relationship between umbral core intensity and spot area has not changed between the two cycles.

We find no evidence for a systematic increase in sunspot intensity during cycle 23 , in disagreement with the findings of Penn \& Livingston (2006) and Livingston et al. (2012). SFO data show that the umbral core intensity is almost constant between 1996 and 2012, when spots of all sizes are included. There is less than $1 \%$ change over the $17 \mathrm{yr}$ covering cycle 23 and the early part of cycle 24 in SFO data compared to the $1.8 \%$ decrease per year reported by Penn \& Livingston (2006). Our results agree with the umbral intensity observations from the higher resolution telescope CFDT2 in operation at SFO since 1992 (Wesolowski et al. 2008) and with the analysis of MDI spot umbrae (Mathew et al. 2007).

The decrease in darkness found by Livingston et al. (2012) can be explained by selection effects and by the small number of measures at the beginning of their data set. The lack of weak magnetic field and faint spots in 1998-2002 indicates that small spots and pores were not included in their early measurements. As solar activity declined, more and more smaller spots were included, and in recent years spots of all sizes have been included. This biased their data set toward stronger fields and darker umbrae during the rise and maximum of cycle 23 . Because of the low solar activity at the end of cycle 23 and beginning of cycle 24 , the latest data are biased toward weaker fields.

When we performed a fit to the SFO observations covering cycle 23 and the rise of cycle 24 excluding small spots during the first years of cycle 23, we found an increase in the intensity of umbrae with time that disappears when small spots are consistently included during all phases of the cycle. We thus conclude that the decrease in umbral darkness found by Livingston et al. (2012) is likely the result of how spots were selected and does not necessarily imply a change in the physical nature of spots. The trend in their data is consistent with the observation that larger spots are, on average, darker and have stronger fields.

We thank Rebecca Centeno for carefully reading the manuscript. We acknowledge support from the NSF/ATM0848518, NASA/NNX11AB51G, and NASA/NNX11AK46G grants and from CSUN and HAO. The National Center for Atmospheric Research is sponsored by the National Science Foundation.

\section{REFERENCES}

Chapman, G. A., Cookson, A. M., Dobias, J. J., Preminger, D. G., \& Walton, S. R. 2004, AdSpR, 34, 262

Chapman, G. A., Cookson, A. M., Dobias, J. J., \& Walton, S. R. 2001, ApJ, 555,462

Chapman, G. A., Herzog, A. D., Lawrence, J. K., et al. 1992, JGR, 97, 8211

de Toma, G., Chapman, G. A., Cookson, A. M., \& Preminger, D. G. 2013, ApJ, 770,89

Leka, K. D., \& Skumanich, A. 1998, ApJ, 507, 454

Livingston, W. C., \& Penn, M. J. 2009, EOSTr, 90, 30

Livingston, W. C., Penn, M. J., \& Svalgaard, L. 2012, ApJL, 757, L8

Mathew, S. K., Martínez-Pillet, V., Solanki, S. K., \& Krivova, N. A. 2007, A\&A, 465, 291

Nagovitsyn, Y. A., Pevtsov, A. A., \& Livingston, W. C. 2012, ApJL, 758, L20

Norton, A. A., \& Gilman, P. A. 2004, ApJ, 603, 348

Penn, M. J., \& Livingston, W. C. 2006, ApJL, 649, L45

Penn, M. J., \& Livingston, W. C. 2010, in IAU Symp. 273, The Physics of Sun and Star Spots, ed. D. Choudhary \& K. Strassmeier (Cambridge: Cambridge Univ. Press), 126

Penn, M. J., \& MacDonald, R. K. D. 2007, ApJL, 662, L123

Pevtsov, A. A., Nagovitsyn, Y. A., Tlatov, A. G., \& Rybak, A. L. 2011, ApJL, 742, L36

Preminger, D. G., Walton, S. R., \& Chapman, G. A. 2001, SoPh, 202, 53

Rezaei, R., Beck, C., \& Schmidt, W. 2012, A\&A, 541, A60

Schad, T. A., \& Penn, M. J. 2010, SoPh, 262, 19

Walton, S. R., Chapman, G. A., Cookson, A. M., Dobias, J. J., \& Preminger, D. G. 1998 , SoPh, 179, 31

Walton, S. R., \& Preminger, D. G. 1999, ApJ, 514, 959

Wesolowski, M. J., Walton, S. R., \& Chapman, G. A. 2008, SoPh, 248, 141 